\title{
A New Rateless Coded Cooperation Scheme for Multiple Access Channels
}

\author{
Wei Chen, and Wen Chen \\ Wireless Network Transmission Laboratory \\ Shanghai Jiao Tong University, China \\ Email: $\{$ chx_ufo;wenchen $\} @$ sjtu.edu.cn
}

\begin{abstract}
The conventional rateless coded cooperation (RCC) operates in full-duplex and non-orthogonal multiples access channel (MAC) aiming to enlarge outage capacity with multiplexed Raptor code of high complexity. In this paper, we develop a new RCC scheme using rateless code, which operates in the practical half-duplex or orthogonal channel aiming to achieves coding gain in high SNR region with only one raptor code. By deriving the outage probability, we found that our scheme outperforms the conventional RCC scheme. Simulation results also validate the theoretical prediction.
\end{abstract}

\section{INTRODUCTION}

In many wireless applications, wireless transmitters may not be able to support multiple antennas due to size, complexity, power, or other constraints. In the face of this limitation, cooperation between pairs of users has been discussed as a means to provide transmit diversity [1], [2]. In these cooperative schemes, diversity is achieved by signaling scheme that allows two single-antenna users to each send their information using both of their antennas. The basic approach to the cooperation has been for a mobile to listen to a partner's transmission, and in a different time or frequency slot to transmit either an amplified version of the received signal (amplify-and-forward) or a decoded version of the received signal (decode-andforward) to the destination [3].

In [4], Hunter and Nosratinia introduced a channel-coding method to achieve cooperative diversity called coded cooperation (CC). The outage behavior of this cooperative protocol is illustrated in [5], which confirms that full diversity can be achieved by coded cooperation. It also shows that coded cooperation enjoys improved performance compared to many cooperation protocols, such as Amplify-and-Forward (AF), Selection Decode-and-Forward (SDF), and Space-time cooperation. The bit-error rate (BER) performance of coded cooperation is also shown to be competitive by employing ratecompatible punctured convolutional (RCPC) codes [6]. In [8], turbo code is used to implement coded cooperation.

Nevertheless, the conventional coded cooperation can not make efficient use of the system resources. In those schemes, the code rate and cooperation level is fixed before transmission. Whether one user will be cooperated is determined by the channel realization from him to his partner. The user can be cooperated only if the channel gain is large enough. In [15], [16], [17], a cooperation scheme using rateless codes is proposed to improve outage capacity in the low power regime, which is called rateless coded cooperation (RCC). The key idea of RCC is to adapt the cooperation level to the channel realization between users and use multiplexed Raptor codes to transmit the information of both Users in the cooperative phase. The channels considered in those schemes is non-orthogonal MAC channels, which can be generalized to orthogonal MAC channels straightly. However, those schemes did not sufficiently exploit the potential of rateless codes. In this paper, we propose a new RCC scheme working under orthogonal MAC channels. Our work is different from the conventional RCC in four aspects.

- The aim of conventional RCC is to enlarge the outage capacity in the low power regime. However, our aim is to achieve coding gain in high SNR regime.

- The users of conventional RCC operate in full-duplex and non-orthogonal MAC channels while users in our scheme operate in half-duplex and orthogonal channels.

- The conventional RCC exploits multiplexed Raptor codes in the cooperative phase, which requires high complexity decoding at the base station. The simplification of multiplexed Raptor codes will suffer performance degradation inevitably. In contrast, our scheme requires only one Raptor code for each user, which reduces the encoding and decoding complexity.

- The conventional RCC employs ACK between each users, however, our scheme employ ACK from the destination to both users. And the transmission schedule between our RCC scheme and the conventional RCC scheme is totally different.

We will describe the system model in section II and the transmission schedule in section III. The outage behavior of the proposed scheme will be analyzed in section IV. In section $\mathrm{V}$, we will show the simulation results. And the conclusion will be made in section VI.

\section{SySTEM MODEL}

As in [4], we consider the cooperative multiple access channel, where two users transmitting data to a single receiver cooperate by alternatively being the relay for the other user, as indicated in Fig. 1. The users transmit on orthogonal channels (e.g., TDMA, CDMA, or FDMA). The receivers have channel state information, but the transmitters do not. We model the channel as frequency-non selective block fading channels whose fading coefficient $h$ remains constant in one 


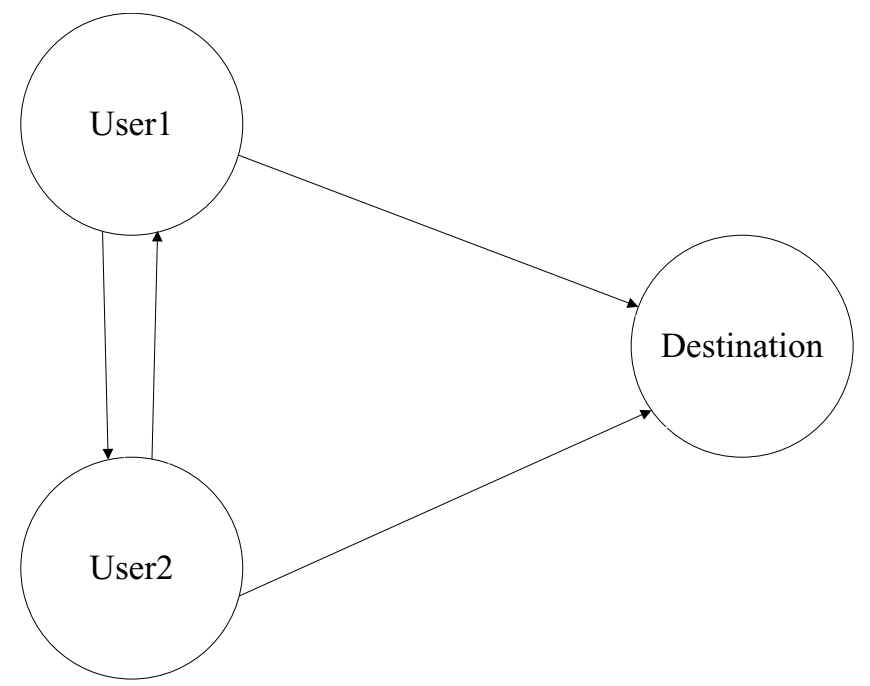

Fig. 1. A Cooperative Multiple Access Channel (MAC). Arrows between two nodes illustrate that both nodes communicate between each other.

block, but changes independently between successive blocks. The fading coefficient $h$ is a zero-mean, circularly symmetric complex Gaussian random variable with unit variance. So the magnitude $|h|$ is Rayleigh distributed, and the channel power $\gamma=|h|^{2}$ is exponentially distributed with probability density function (pdf) with parameter $1 / \Gamma$, where $\Gamma$ denotes the mean value of SNR over the fading and accounts for the combination of transmit power, large-scale path loss and shadowing effects. The outage probability is thus defined as

$$
\begin{aligned}
P_{\text {out }} & =\operatorname{Pr}\left\{\gamma<2^{R}-1\right\}=\int_{0}^{2^{R}-1} p_{\gamma}(\gamma) d \gamma \\
& =\int_{0}^{2^{R}-1} \frac{1}{\Gamma} \exp \left(-\frac{\gamma}{\Gamma}\right) d \gamma \\
& =1-\exp \left(-\frac{2^{R}-1}{\Gamma}\right) .
\end{aligned}
$$

To simplify the notation, we define $C(x) \equiv \log _{2}(1+x)$.

In our scheme, rateless codes such as LT code or Raptor code [13], [14] are introduced instead of RCPC code [6] or turbo code [8] in coded cooperation. One of the benefits of rateless codes is that it generates the code bit on the fly and can adapt the code rate to the channel condition. On fading channels or fading relay channels, rateless codes have been shown to be very efficient in [9], [11]. Here, we focus on TDMA systems where users operate in half-duplex. The generalization to FDMA or CDMA system is trivial. We also employ an 1-bit ACK from the destination to both users. If the destination decodes one of the users' transmission successfully, it will transmit a feedback message to both users. Therefore, both of the users will know whether itself or its partner's transmission has been successfully decoded by destination immediately.

\section{TRANSMISSION SCHEDULING}

We denote the period that User 1 and User 2 both transmit a codeword cooperatively as a transmission slot. In each transmission slot, User 1 transmit first, and then User 2 transmit. Despite infinitely long code-stream can be generated by rateless codes, we assume each user will stop transmission after $N$ symbols have been transmitted in one transmission slot. Thus the symbols transmitted in our scheme is less than or equal to conventional coded cooperation.

In the proposed scheme, User 1 start to transmit when User 2 stop its transmission. First, User 1 transmits its own information via rateless codes until the ACK from the destination is received or $N$ symbols are transmitted. If the destination successfully decode User 1's message before $N$ symbols is transmitted, User 1 will try to help User 2 with the symbols left. User 1 will check whether User 2's information in the last transmission slot is decoded successfully by destination (according to ACK) and itself. If User 1 decodes successfully but the destination failed, User 1 will transmit rateless code symbols regenerated from User 2's information. Transmission won't stop until getting the ACK from the destination or totally $N$ symbols are transmitted. The flow-process diagram is depicted in Fig. 2, and the transmission schedule is depicted in Fig. 3.

User 1 and User 2 have almost the same transmission scheduling, and the only difference is that User 2 cooperates with User 1 to transmit User 1's information in the same transmission slot. It is because that User 1 transmits first in each slot. So we just consider the case for User 1 .

Thus the outage events for User 1 in our scheme is

or

$$
\begin{aligned}
& C\left(\gamma_{1, d}\right)<R \\
& C\left(\gamma_{1,2}\right)<R
\end{aligned}
$$

where $\gamma_{1,2}, \gamma_{1, d}$ and $\gamma_{2, d}$ denote the instantaneous SNRs of the channel User $1 \rightarrow$ User2, the channel User $1 \rightarrow$ destination and the channel User $2 \rightarrow$ destination respectively. There are several reasons that our RCC scheme outperforms the $\mathrm{CC}$ and the conventional RCC given as follows.

- Mutual information accumulation is shown to be better than energy accumulation in repetition based schemes [12]. From this point of view, we can see that the conventional coded cooperation uses both mutual information accumulation and energy accumulation. Instead, only mutual information accumulation is used in our scheme.

- If the inter-user channels are perfect, both the $\mathrm{CC}$ and the conventional RCC will have the same Outage behavior. The Outage event is $\left\{C\left(\gamma_{1, d}\right)+C\left(\gamma_{2, d}\right)<2 R\right\}$. However, the outage event of our scheme is $\left\{C\left(\gamma_{1, d}\right)<\right.$ $\left.R, C\left(\gamma_{1, d}\right)+C\left(\gamma_{2, d}\right)<2 R\right\}$ for User 1 and $\left\{C\left(\gamma_{2, d}\right)<\right.$ $\left.R, C\left(\gamma_{1, d}\right)+C\left(\gamma_{2, d}\right)<2 R\right\}$ for User 2 , which is smaller than $\mathrm{CC}$ or conventional RCC. This is because that in 


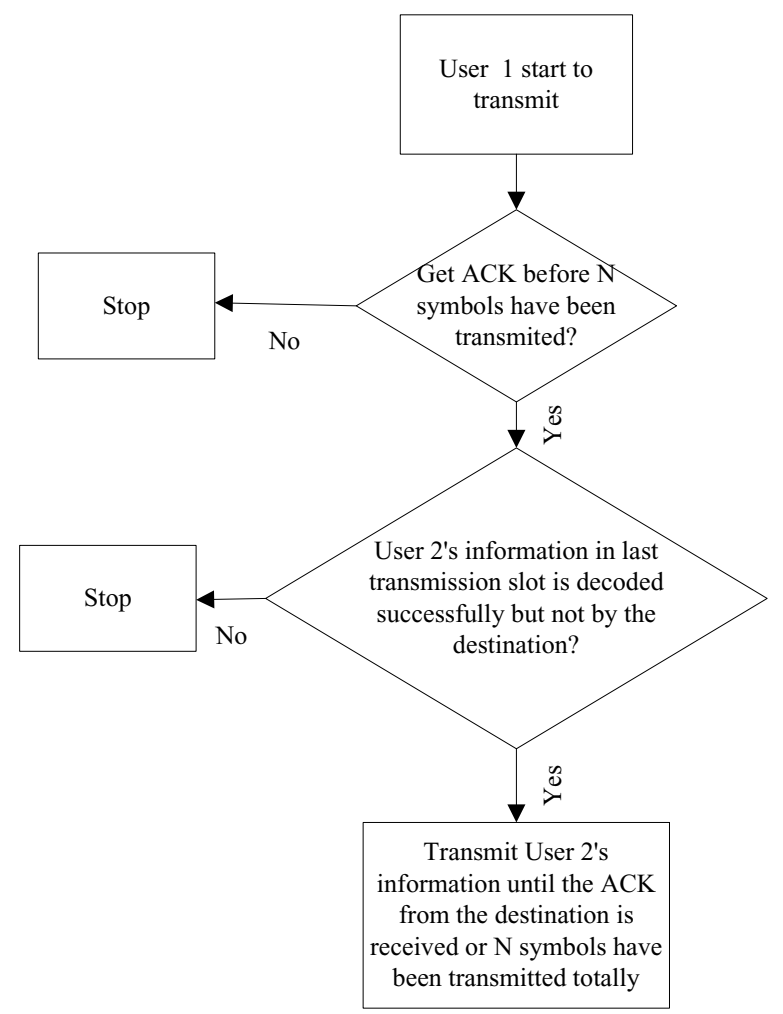

Fig. 2. The flow Chart of the proposed RCC in User 1.

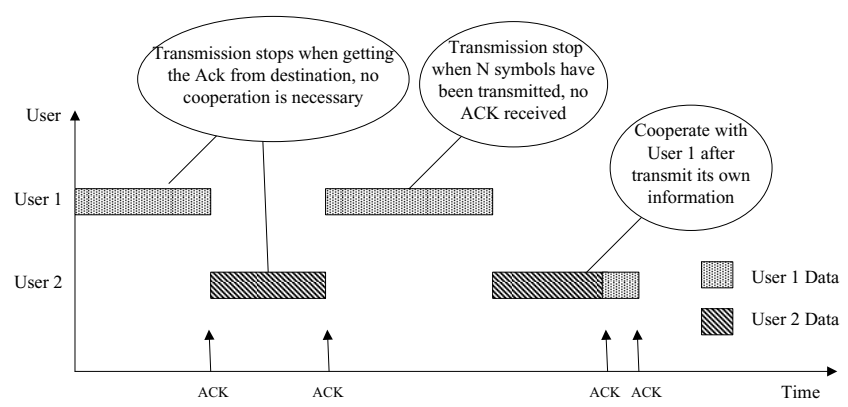

Fig. 3. Transmission scheduling

our scheme one user won't cooperate with his partner until the destination decode his information successfully, which can avoid the user being dragged down to outage by his partner while the user himself is able to transmit his information. We call this "dragging effect".

- In the conventional RCC, both users operate in full duplex and transmit the information simultaneously. The cooperative phase won't start until both of the users decode each other successfully. This will cause information waste when the destination has decoded one user's information. But this user can't help his partner. In our scheme, however, we stagger the two users' transmission in time domain and each user cooperates by relaying his partner's information transmitted in the last time slot. Thus users operate in half duplex and one user can cooperate with his partner at any time during his transmission. We employ
ACK from the destination to both users, which helps to avoid the information waste totally.

- In addition, our scheme is more suitable for unequal mean SNR on the uplink channel, and can even work well in the scenario that User 1 and User 2 have different code rates.

\section{Outage Probability Analysis}

In this section, we will derive the outage probability of the proposed rateless code based cooperation scheme. For simplicity we focus on the outage behavior for User 1. As indicated in the last section, the outage probability of User 1 can be expressed as

$$
\begin{aligned}
P_{\text {out }, 1, r c c}= & \operatorname{Pr}\left\{C\left(\gamma_{1,2}\right)>R\right\} \\
& \cdot \operatorname{Pr}\left\{C\left(\gamma_{1, d}\right)<R, C\left(\gamma_{1, d}\right)+C\left(\gamma_{2, d}\right)<2 R\right\} \\
& +\operatorname{Pr}\left\{C\left(\gamma_{1,2}\right)<R\right\} \cdot \operatorname{Pr}\left\{C\left(\gamma_{1, d}\right)<R\right\} . \quad \text { (4) }
\end{aligned}
$$

From (1), we can get

$$
\begin{aligned}
& \operatorname{Pr}\left\{C\left(\gamma_{1,2}\right)>R\right\}=\exp \left(-\frac{2^{R}-1}{\Gamma_{1,2}}\right), \\
& \operatorname{Pr}\left\{C\left(\gamma_{1,2}\right)<R\right\}=1-\exp \left(-\frac{2^{R}-1}{\Gamma_{1,2}}\right), \\
& \operatorname{Pr}\left\{C\left(\gamma_{1, d}\right)<R\right\}=1-\exp \left(-\frac{2^{R}-1}{\Gamma_{1, d}}\right),
\end{aligned}
$$

where $\Gamma_{1,2}$ and $\Gamma_{1, d}$ are the average SNRs of the channel from User 1 to User 2, and the channel from User 1 to destination. Moreover, we have

$$
\begin{aligned}
\operatorname{Pr} & \left\{\mathbb{C}\left(\gamma_{1, d}\right)<R, C\left(\gamma_{1, d}\right)+C\left(\gamma_{2, d}\right)<2 R\right\} \\
= & \int_{0}^{2^{R}-1} \int_{0}^{f\left(\gamma_{1, d}\right)} \Phi\left(\gamma_{1, d}, \gamma_{2, d}, \Gamma_{1, d}, \Gamma_{2, d}\right) d \gamma_{2, d} d \gamma_{1, d} \\
= & \int_{0}^{2^{R}-1} \frac{1}{\Gamma_{1, d}} \exp \left(-\frac{\gamma_{1, d}}{\Gamma_{1, d}}\right)\left(1-\exp \left(-\frac{f\left(\gamma_{1, d}\right)}{\Gamma_{2, d}}\right)\right) d \gamma_{1, d} \\
= & 1-\exp \left(-\frac{2^{R}-1}{\Gamma_{1, d}}\right) \\
& -\int_{0}^{2^{R}-1} \exp \left(-\frac{\gamma_{1, d}}{\Gamma_{1, d}}-\frac{f\left(\gamma_{1, d}\right)}{\Gamma_{2, d}}\right) d \gamma_{1, d},
\end{aligned}
$$

where

$$
\begin{aligned}
\Phi\left(\gamma_{1, d}, \gamma_{2, d}, \Gamma_{1, d}, \Gamma_{2, d}\right) & =\frac{1}{\Gamma_{1, d}} \exp \left(-\frac{\gamma_{1, d}}{\Gamma_{1, d}}\right) \frac{1}{\Gamma_{2, d}} \exp \left(-\frac{\gamma_{2, d}}{\Gamma_{2, d}}\right) \\
f\left(\gamma_{1, d}\right) & \equiv 2^{2 R-\log _{2}\left(1+\gamma_{1, d}\right)}-1
\end{aligned}
$$

Then, the outage probability can be derived from (4), (5) and (6). There is no closed form of the outage probability. But it can be demonstrated by numerical methods. Nevertheless, we can get asymptotic expression of outage probability by Taylor series expansion and analyze the diversity order of the proposed system. We now assume that the user transmit power goes to infinity. Thus the mean SNR of the uplink channel and 
the inter-user channel will go to infinity at the same order. Using Taylor series expansion, we have

$$
\begin{aligned}
\operatorname{Pr}\left\{C\left(\gamma_{1,2}\right)<R\right\} & =1-\exp \left(-\frac{2^{R}-1}{\Gamma_{1,2}}\right) \\
& =\frac{2^{R}-1}{\Gamma_{1,2}}+O\left(\frac{1}{\Gamma^{2}}\right), \\
\operatorname{Pr}\left\{C\left(\gamma_{1, d}\right)<R\right\} & =1-\exp \left(-\frac{2^{R}-1}{\Gamma_{1, d}}\right) \\
& =\frac{2^{R}-1}{\Gamma_{1, d}}+O\left(\frac{1}{\Gamma^{2}}\right), \\
\operatorname{Pr}\left\{C\left(\gamma_{1,2}\right)>R\right\} & =\exp \left(-\frac{2^{R}-1}{\Gamma_{1,2}}\right) \\
& =1+O\left(\frac{1}{\Gamma}\right) .
\end{aligned}
$$

So we have

$$
\begin{aligned}
\operatorname{Pr}\left\{C\left(\gamma_{1,2}\right)\right. & <R\} \cdot \operatorname{Pr}\left\{C\left(\gamma_{1, d}\right)<R\right\} \\
& =\frac{\left(2^{R}-1\right)^{2}}{\Gamma_{1,2} \Gamma_{1, d}}+O\left(\frac{1}{\Gamma^{3}}\right) .
\end{aligned}
$$

We can also use Taylor series expansion to calculate (6) and get

$$
\begin{array}{r}
\operatorname{Pr}\left\{C\left(\gamma_{1, d}\right)<R, C\left(\gamma_{1, d}\right)+C\left(\gamma_{2, d}\right)<2 R\right\} \\
=\frac{R 2^{2 R} \ln 2-2^{R}+1}{\Gamma_{1, d} \Gamma_{2, d}}+O\left(\frac{1}{\Gamma^{3}}\right) .
\end{array}
$$

Finally we obtain the asymptotic outage probability expression for User 1 as

$$
P_{\text {out }, 1, r c c}=\frac{\left(2^{R}-1\right)^{2}}{\Gamma_{1,2} \Gamma_{1, d}}+\frac{R 2^{2 R} \ln 2-2^{R}+1}{\Gamma_{1, d} \Gamma_{2, d}}+O\left(\frac{1}{\Gamma^{3}}\right) .
$$

We can observe that as the mean SNR tends to infinity, the outage probability decays at the order $O\left(S N R^{-2}\right)$, which implies that the proposed new scheme can achieve full diversity.

We can also show that the proposed scheme outperforms the conventional coded cooperation by comparing the outage probability at high SNR. By [5], the outage probability of CC scheme is

$$
P_{\text {out }, 1, c c}=\frac{\left(2^{R / \alpha}-1\right)^{2}}{\Gamma_{1,2} \Gamma_{1, d}}+\frac{\Lambda(R, \alpha)}{\Gamma_{1, d} \Gamma_{2, d}}+O\left(\frac{1}{\Gamma^{3}}\right),
$$

where

$\Lambda(R, \alpha)= \begin{cases}\left(\frac{\alpha}{1-2 \alpha}\right) 2^{R / \alpha}-\left(\frac{1-\alpha}{1-2 \alpha}\right) 2^{R /(1-\alpha)}+1, & \alpha \neq 1 / 2, \\ R \cdot 2^{2 R+1} \cdot \ln 2-2^{2 R}+1, & \alpha=1 / 2,\end{cases}$

and the $0<\alpha<1$ describes the cooperation level in CC scheme. Since $2^{R / \alpha}>2^{R}$, we have

$$
\frac{\left(2^{R / \alpha}-1\right)^{2}}{\Gamma_{1,2} \Gamma_{1, d}}>\frac{\left(2^{R}-1\right)^{2}}{\Gamma_{1,2} \Gamma_{1, d}} .
$$

We can also prove that

$$
\Lambda(R, \alpha) \geq \Lambda\left(R, \frac{1}{2}\right) \geq R 2^{2 R} \ln 2-2^{R}+1 .
$$

Thus (12) and (13) show that the outage probability of RCC scheme is less than that of CC scheme at high SNR regime. Since the conventional RCC scheme has the same outage probability as the $\mathrm{CC}$ scheme at high SNR regime, the proposed RCC scheme outperforms the $\mathrm{CC}$ scheme and the conventional RCC scheme at high SNR regime in terms of outage probability.

\section{Simulation Results}

In this section, we give the comparison of outage probability for the proposed RCC scheme and the previous coded cooperation scheme. In Fig. 4 , we set $R=1 / 2 b / s / H z$. It shows outage probability versus mean uplink SNR $\left(\Gamma_{1, d}=\Gamma_{2, d}\right)$ for various conditions of the inter-user channel. The different sets of curves correspond to the inter-user mean SNR equal to the mean uplink SNR, 10dB less than the mean uplink SNR, and equal to $\infty$. All of the curves in Fig. 4 show that both CC and RCC have the diversity order two. We can observe that the coding gain of RCC is relatively large when the inter-user mean SNR is equal to the uplink mean SNR.

In Fig. 5, we compares outage probability versus mean uplink SNR for the proposed RCC, the conventional RCC, CC, AF, SDF. We consider rate $R=1 / 2$, with all channels having equal mean SNR. we can see that the proposed RCC scheme outperforms any other cooperation scheme and the performance gain is quite large. However, to get such performance gain is impossible in practice because it is impossible to generate "universal" rateless codes that are simultaneously perfect at all possible rates [18]. But we can find rateless codes with a bounded overhead compared with perfect codes as indicated in [12]. We can also observe that the outage probabilities of the $\mathrm{CC}$ shcme and the conventional RCC scheme almost meet at high SNR regime.

Fig. 6 shows outage probability versus uplink SNR for unequal mean uplink SNR $\left(\Gamma_{1, d} \neq \Gamma_{2, d}\right)$. The $x$-axis is the mean uplink SNR for User 2, $\Gamma_{2, d}$. In this case, User 1 has a better uplink channel, with mean SNR $\Gamma_{1, d}=\Gamma_{2, d}+10 d B$. The mean inter-user SNR is equal to $\Gamma_{2, d}$. We can observe that both users can get about $1 \mathrm{~dB}$ performance gain and the difference of outage probability between each user is smaller by using RCC instead of CC, which means RCC helps to allocate the system resource more efficiently.

\section{CONCLUSION}

In this paper, we develop a new RCC scheme using rateless code, which operates in the practical half-duplex or orthogonal channel aiming to achieves coding gain in high SNR region with only one raptor code. By deriving the outage probability, we found that our scheme outperforms the conventional RCC scheme. Simulation results also validate the theoretical prediction.

\section{ACKNOWLEDGEMENT}

This work is supported by NSF China \#60972031, by SEU SKL project \#W200907, by Huawei Funding \#YJCB2009024WL and \#YJCB2008048WL, and by National 973 project \#2009CB824900. 


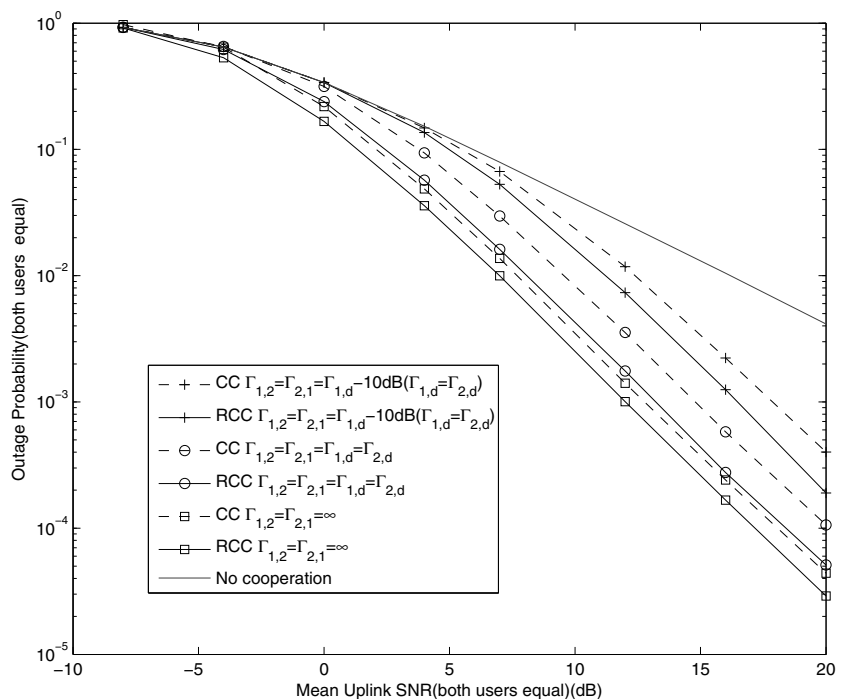

Fig. 4. Outage probability versus SNR for rate $R=1 / 2 b / s / H z$. Various sets of curves correspond to the inter-user channel mean SNR equanl to the mean uplink SNR, $10 d B$ less than the mean uplink SNR, and equal to $\infty$; e.g., a noiseless inter-user channel.

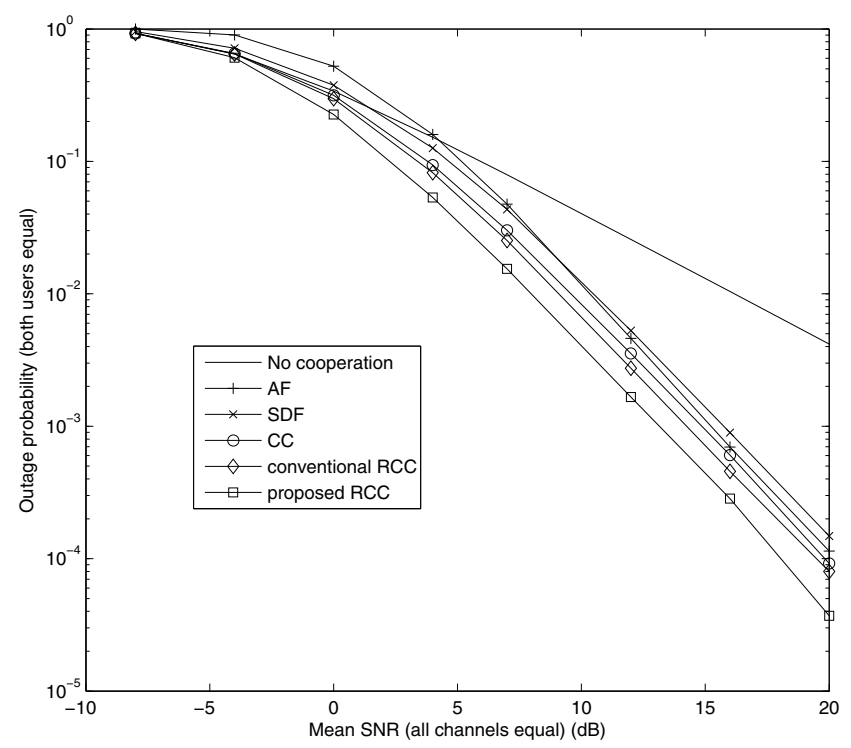

Fig. 5. Outage probability versus $S N R$ for rate $\mathrm{R}=1 / 2 \mathrm{~b} / \mathrm{s} / \mathrm{Hz}$. All channels have equal mean SNR. Comparison of Rateless Coded cooperation, Coded cooperation, amplify-and-forward, and selection decode-and-forward.

\section{REFERENCES}

[1] A. Sendonaris, E. Erkip, and B. Aazhang, "User cooperation diversityPart I: System Description”, IEEE Trans. Commun., vol. 51, no. 11, pp. 1927 - 1938, November 2003.

[2] A. Sendonaris, E. Erkip, and B. Aazhang, "User cooperation diversityPart II: Implemetation aspects and performance analysis", IEEE Trans. Commun., vol. 51, no. 11, pp. 1939-1948, November 2003.

[3] A. Nosratinia, T. Hunter, and A. Hedayat, "Cooperative communication in wireless networks", IEEE Commun. Mag., vol. 42, no. 10, pp. 68 - 73, October 2004.

[4] T. Hunter and A. Nosratinia, "Cooperative diversity through coding",

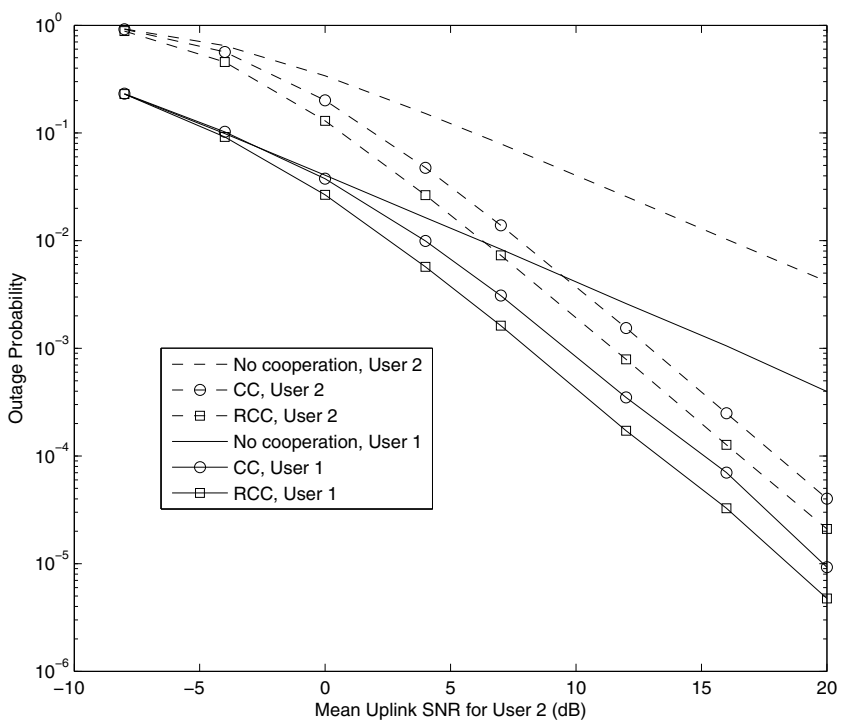

Fig. 6. Outage probability versus $S N R$ for rate $R=1 / 2 b / s / H z$ and unequal mean SNR for the uplink channels. The mean SNR of the inter-user channel is equal to that of User 2. The mean SNR of User 1's uplink channel is 10 dB higher.

IEEE International Symposium on Information Theory(ISIT), Laussane, Switzerland, July 2002, p.220.

[5] T. Hunter, S. Sanayei, and A. Nosratinia, "Outage Analysis of Coded Cooperation", IEEE Transactions on Information Theory, vol. 52, no. 2, pp. 375 - 391, February 2006.

[6] T. Hunter, and A. Nosratinia, "Diversity through Coded Cooperation", IEEE Transactions on Wireless Communications, vol. 5, no. 2, pp. 283 289, February 2006.

[7] T. J. Richardson and R. L. Urbanke, Modern Coding Theory, Cambridge University Press, 2008.

[8] M. Janani, A. Hedayat, T. E. Hunter and A. Nosratinia, "Coded Cooperation in Wireless Communications: Space-Time Transmission and Iterative Decoding”, IEEE Transactions on Wireless Communications, vol. 52, no. 2, pp. 362 - 371, February 2004.

[9] J. Castura and Y. Mao, "Rateless Coding over Fading Channels", IEEE Communications Letters, vol. 10, no. 1, pp. 46-48, January 2006.

[10] J. Castura and Y. Mao, "Rateless Coding for Wireless Relay Channels" IEEE Transactions on Wireless Communications, vol. 6, no. 5, pp. 16381642, May 2007.

[11] X. Liu and T. J. Lim, "Fountain Codes over Fading Relay Channels", IEEE Transactions on Wireless Communications, vol. 8, no. 6, pp. 32783287, JUNE 2009.

[12] A. F. Molisch, N. B. Mehta, J. S. Yedidia and J. Zhang, "Performance of Fountain Codes in Collaborative Relay Networks", IEEE Transactions on Wireless Communications, vol. 6, no. 11, pp. 4108-4119, NOVEMBER 2007.

[13] M. Luby, "LT codes," in Proc. 43rd Annual IEEE Symposium Foundations Computer Science (FOCS), pp. 271-280, 2002.

[14] A. Shokrollashi, "Raptor codes," IEEE Trans. Inform. Theory, vol. 52, no.6, pp. 2551-2567, JUNE 2006.

[15] Z. Yang and A. Host-Madsen, "Rateless coded cooperation for multiple access channels in the low power regime," Proc ISIT 06, Seattle, WA, July 2006.

[16] M. Uppal, A. Host-Madsen, and Z. Xiong, "Practical rateless cooperation in multiple access channels using multiplexed Raptor codes," Proc. ISIT 06, Nice, France, June 2006.

[17] M. Uppal, A. Host-Madsen and Z. Xiong,"Cooperation in the MAC Channel Using Frequency Division Multiplexing,"Proc. ISIT 09, Seoul, Korea, July 2009.

[18] O. Etasami, M. Molkaraie, and A. Shokrollahi, "Raptor codes on symmetric channels," Proc. ISIT, 2004, p.38. 